HIP-2003-57/TH

KEK-TH-926

KIAS-P03081

VPI-IPPAP-03-18

\title{
Lifting degeneracies in the oscillation parameters by a neutrino factory
}

\author{
Mayumi Aoki ${ }^{1,2 *}$, Kaoru Hagiwara ${ }^{2}$, and Naotoshi Okamura ${ }^{3,4 \dagger}$ \\ ${ }^{1}$ Helsinki Institute of Physics, P.O.Box 64 FIN-00014, University of Helsinki, Finland \\ ${ }^{2}$ Theory Group, KEK, Tsukuba, Ibaraki 305-0801, Japan \\ ${ }^{3}$ IPPAP, Physics Department, Virginia Tech. Blacksburg, VA 24061, USA \\ ${ }^{4}$ Korea Institute for Advanced Study, 207-43 Cheongnyangni 2dong \\ Dongdaemun-gu, Seoul 130-722, Republic of Korea
}

\begin{abstract}
We study the potential of a very long baseline neutrino oscillation experiment with a neutrino factory and a large segmented water-Čerenkov calorimeter detector in resolving the degeneracies in the neutrino oscillation parameters; the sign of the larger mass-squared difference $\delta m_{13}^{2}$, the sign of $\left|U_{\mu 3}\right|^{2}\left(\equiv \sin ^{2} \theta_{\text {ATM }}\right)-1 / 2$, and a possible two-fold ambiguity in the determination of the CP phase $\delta_{\text {MNS }}$. We find that the above problems can be resolved even if the particle charges are not measured. The following results are obtained in our exploratory study for a neutrino factory which delivers $10^{21}$ decaying $\mu^{+}$and $\mu^{-}$at $10 \mathrm{GeV}$ and a $100 \mathrm{kton}$ detector which is placed $2,100 \mathrm{~km}$ away and is capable of measuring the event energy and distinguishing $e^{ \pm}$from $\mu^{ \pm}$, but not their charges. The sign of $\delta m_{13}^{2}$ can be determined for $4\left|U_{e 3}\right|^{2}\left(1-\left|U_{e 3}\right|^{2}\right) \equiv$ $\sin ^{2} 2 \theta_{\mathrm{RCT}} \gtrsim 0.008$. That of $\sin ^{2} \theta_{\mathrm{ATM}}-1 / 2$ can be resolved for $\sin ^{2} 2 \theta_{\mathrm{ATM}}=0.96$ when $\sin ^{2} 2 \theta_{\mathrm{RCT}} \gtrsim 0.06$. The CP-violating phase $\delta_{\mathrm{MNS}}$ can be uniquely constrained for $\sin ^{2} 2 \theta_{\mathrm{RCT}} \gtrsim 0.02$ if its true value is around $90^{\circ}$ or $270^{\circ}$, while it can be constrained for $\sin ^{2} 2 \theta_{\mathrm{RCT}} \gtrsim 0.03$ if its true value is around $0^{\circ}$ or $180^{\circ}$.
\end{abstract}

PACS : 14.60.Lm, 14.60.Pq, 01.50.My

Keywords: neutrino oscillation experiment, long base line experiments, future plan

*mayumi.aoki@kek.jp

†nokamura@kias.re.kr 
Atmospheric neutrino observation at Super-Kamiokande (SK) [1] and the K2K experiment [2] established $\nu_{\mu}$ oscillation with a close-to-maximal mixing $\sin ^{2} 2 \theta_{\text {ATM }}>0.9$ and $\delta m_{\mathrm{ATM}}^{2} \sim(1.3-3.0) \times 10^{-3} \mathrm{eV}^{2}$. The MSW large-mixing-angle (LMA) solution [3, 4] of the solar-neutrino deficit problem [5, 6, 7] has been established by KamLAND [8] and by the improved measurement by SNO [7]. The best fit values are $\sin ^{2} 2 \theta_{\text {sou }}=0.82$ and $\delta m_{\mathrm{SOL}}^{2}=7.1 \times 10^{-5} \mathrm{eV}^{2}$. The CHOOZ [9] and Palo Verde [10] reactor experiments give upper bounds on the third mixing angle of the three-neutrino model; $\sin ^{2} 2 \theta_{\text {RCT }} \lesssim 0.1(0.2)$ for $\delta m_{\mathrm{ATM}}^{2} \sim 3.0(2.0) \times 10^{-3} \mathrm{eV}^{2}$.

In the three-neutrino model, the present neutrino oscillation experiments constrain $U_{e 2}$, $U_{e 3}$, and $U_{\mu 3}$ matrix elements of the lepton-flavor mixing matrix $U_{\text {MNS }}$ (Maki-NakagawaSakata (MNS) [11]) :

$$
\begin{aligned}
\left|U_{\mu 3}\right|^{2} & \equiv \sin ^{2} \theta_{\mathrm{ATM}}=\left(1 \pm \sqrt{1-\sin ^{2} 2 \theta_{\mathrm{ATM}}}\right) / 2, \\
\left|U_{e 2}\right|^{2} & =\left(1-\left|U_{e 3}\right|^{2}-\sqrt{\left(1-\left|U_{e 3}\right|^{2}\right)^{2}-\sin ^{2} 2 \theta_{\mathrm{SOL}}}\right) / 2, \\
\left|U_{e 3}\right|^{2} & =\left(1-\sqrt{1-\sin ^{2} 2 \theta_{\mathrm{RCT}}}\right) / 2,
\end{aligned}
$$

and the mass-squared differences, $\delta m_{\mathrm{SOL}}^{2}=\left|\delta m_{12}^{2}\right| \ll\left|\delta m_{13}^{2}\right|=\delta m_{\mathrm{ATM}}^{2}$, where $\delta m_{i j}^{2} \equiv$ $m_{j}^{2}-m_{i}^{2}$. The matrix elements $U_{e 2}$ and $U_{\mu 3}$ are taken to be real and non-negative while $U_{e 3}$ is a complex number in our convention [12, 13]. The CP-violating phase $\delta_{\mathrm{MNS}}=-\arg \left(U_{e 3}\right)$, is unconstrained. Note that the solution for $U_{e 2}$ follows from our convention $U_{e 1}>U_{e 2}$ [13], which defines the mass-eigenstate $\nu_{1}$.

The present experiments allow several degeneracies in the neutrino oscillation parameters. First is the sign of the larger mass-squared difference, $\delta m_{13}^{2} \equiv m_{3}^{2}-m_{1}^{2}= \pm \delta m_{\text {Aтм }}^{2}$. The sign of the smaller mass-squared difference is determined as $\delta m_{12}^{2} \equiv m_{2}^{2}-m_{1}^{2}=\delta m_{\text {soL }}^{2}$ by the MSW matter effects in the sun. The mass hierarchy $\delta m_{13}^{2}=\delta m_{\text {ATM }}^{2}$ is called 'normal' and $\delta m_{13}^{2}=-\delta m_{\text {ATM }}^{2}$ is called 'inverted'. The second degeneracy is in the mixing angle $\theta_{\text {AтM }}$. Although the present experiments constrain $\sin ^{2} 2 \theta_{\text {ATM }} \simeq 1, \sin ^{2} \theta_{\text {ATM }}$ has two-fold ambiguity, $\sin ^{2} \theta_{\text {ATM }}-1 / 2= \pm \sqrt{1-\sin ^{2} 2 \theta_{\text {ATM }}} / 2$. Furthermore, there can appear two-fold ambiguity in the future determination of the $\delta_{\mathrm{MNS}}$ phase, between $\delta_{\mathrm{MNS}}$ and $180^{\circ}-\delta_{\mathrm{MNS}}$. Implications of the above degeneracy problems and their partial resolutions have been proposed in [23].

The proposed long-baseline (LBL) neutrino-oscillation experiments [14, 15] will achieve the precision measurements of $\delta m_{\mathrm{ATM}}^{2}$ and $\sin ^{2} 2 \theta_{\mathrm{ATM}}$ by using conventional neutrino beams, which are made from decays of $\pi$ and $K$ that are produced by high-energy proton beams. However, lifting the above eight-fold degeneracy in the neutrino oscillation parameters could remain as the target for the next generation neutrino experiments.

In Ref. [16, we studied the possibility of measuring $\delta_{\mathrm{MNS}}$ in the LBL experiment with 
J-PARC (Japan Proton Accelerator Complex) [17] at Tokai Village and a Megaton-level water-Čerenkov detector, Hyper-Kamiokande (HK) [18]. The distance between Tokai Village and HK is about $295 \mathrm{~km}$. There we found that it is relatively easy to distinguish between $\delta_{\mathrm{MNS}}=90^{\circ}$ and $270^{\circ}$ cases, but that it is difficult to distinguish between $\delta_{\mathrm{MNS}}=0^{\circ}$ and $180^{\circ}$ cases. Those studies, however, assumed that the neutrino mass hierarchy is known to be normal $\left(\delta m_{13}^{2}=\delta m_{\text {ATM }}^{2}\right)$ and also we assumed $\sin ^{2} \theta_{\text {ATM }}=0.5$ as an input.

In Fig प(a), we show the allowed regions of Tokai-to-HK experiment when $\delta_{\mathrm{MNS}}=90^{\circ}$ and the normal hierarchy is assumed. CP-violation can be established at better than $3 \sigma$ level if the neutrino mass hierarchy is known. However, the region encircled by thin lines are allowed if the inverted hierarchy $\left(\delta m_{13}^{2}=-\delta m_{\mathrm{ATM}}^{2}\right)$ is assumed in the analysis of the same data. CP conservation is preferred in the latter case. In Fig प(b), we show the allowed region of the same experiment when $\sin ^{2} \theta_{\mathrm{ATM}}^{\text {true }}=0.45,0.35$. Mirror solutions at around $\sin ^{2} \theta_{\mathrm{ATM}}^{\text {mirror }}=0.55,0.65$, respectively, are clearly seen*.
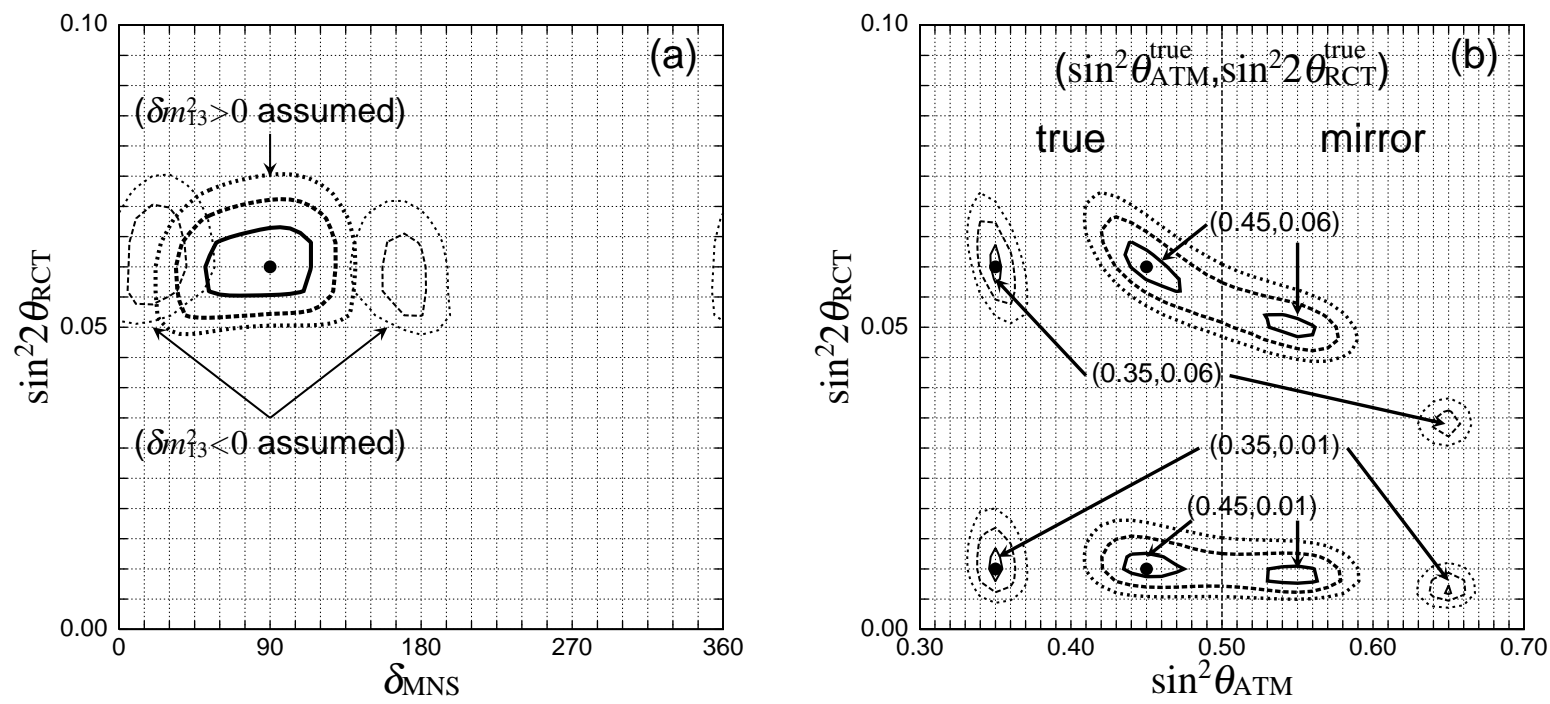

Figure 1: The regions allowed by future LBL experiment between J-PARC at Tokai and HK in the plane of $\delta_{\text {MNS }}$ and $\sin ^{2} 2 \theta_{\text {RCT }}$ (a), and $\sin ^{2} \theta_{\text {ATM }}$ and $\sin ^{2} 2 \theta_{\text {RCT }}$ (b). The assumed experimental conditions are $0.8 \mathrm{Mton} \cdot$ year for $\nu_{\mu} \mathrm{OAB}\left(2^{\circ}\right), 2 \mathrm{Mton} \cdot$ year for $\nu_{\mu} \mathrm{OAB}\left(3^{\circ}\right)$, and 3.2 Mton-year for $\bar{\nu}_{\mu} \mathrm{OAB}\left(2^{\circ}\right) \cdot \chi^{2}$ fittings are performed by assuming the normal and the inverted hierarchies in (a) and the normal hierarchy only in (b).

In this paper, we examine the possibility of a very long-baseline (VLBL) neutrino oscillation experiment with a neutrino factory at J-PARC in resolving the neutrino mass hierarchy, the sign of $\sin ^{2} \theta_{\mathrm{ATM}}-1 / 2$, and the degeneracy in the CP phase $\delta_{\mathrm{MNS}}$. The possibility of a neutrino factory at J-PARC in Tokai is studied in Ref. [20]. For the detector, we assume a 100 kton-level segmented water-Čerenkov calorimeter detector at $L=2,100$

${ }^{*}$ The results in Fig 1 are obtained by using the off-axis beams [15]. Details of the J-PARC-to-HK analyses with the off-axis beams will be reported elsewhere [19]. 
$\mathrm{km}$ away from Tokai. The distance is approximately that between Tokai and Beijing, where strong interests in constructing a large water-Čerenkov detector BAND (Beijing Astrophysics and Neutrino Detector) have been expressed [21]. The BAND detector has a good capability for detecting $\nu_{e}$ charged current $(\mathrm{CC})$ and $\nu_{\mu} \mathrm{CC}$ events with calorimetric energy measurement [21, 22]. In this analysis, we assume that the detector is capable of measuring the event energy and distinguishing $e^{ \pm}$from $\mu^{ \pm}$, but we do not require its charge identification capability.

The physics prospects of VLBL oscillation experiments with a neutrino factory has been studied in the past by assuming that the detector can identify charges [23], and hence the possibility of charge identification at a large water-Cerenkov detector has been investigated 22. We would like to show in this paper that even if a detector is charge blind it could achieve all the goals.

In the neutrino factory, neutrinos are produced from the decay of high energy muons, $\mu^{+} \rightarrow \bar{\nu}_{\mu} \nu_{e} e^{+}$or $\mu^{-} \rightarrow \nu_{\mu} \bar{\nu}_{e} e^{-}$. The same amount of $\bar{\nu}_{\mu}$ and $\nu_{e}\left(\nu_{\mu}\right.$ and $\left.\bar{\nu}_{e}\right)$ are contained in the stored $\mu^{+}\left(\mu^{-}\right)$beam. One of the most significant qualities of the neutrino factory is the well knowledge of neutrino fluxes. Assuming very relativistic muons, the $\bar{\nu}_{\mu}$ and $\nu_{e}$ $\left(\nu_{\mu}\right.$ and $\left.\bar{\nu}_{e}\right)$ fluxes from $\mu^{+}\left(\mu^{-}\right)$beam are expressed as

$$
\begin{aligned}
& \Phi_{\bar{\nu}_{\mu}\left(\nu_{\mu}\right)}=\gamma^{2} \frac{n_{\mu}}{\pi L^{2}} 2 y^{2}\left\{(3-2 y) \mp P_{\mu}(1-2 y)\right\}, \\
& \Phi_{\nu_{e}\left(\bar{\nu}_{e}\right)}=\gamma^{2} \frac{n_{\mu}}{\pi L^{2}} 12 y^{2}\left\{(1-y) \mp P_{\mu}(1-y)\right\}
\end{aligned}
$$

where $\gamma=E_{\mu} / m_{\mu}, y=E_{\nu} / E_{\mu}$ with the energy of the decaying muon $E_{\mu}, P_{\mu}$ is the average muon polarization $\left(P_{\mu}=1\right.$ for right-handed and $P_{\mu}=-1$ for left-handed $\left.\mu^{ \pm}\right)$, and $n_{\mu}$ is the number of the decaying muons. The upper (lower) sign should be taken for $\mu^{+}\left(\mu^{-}\right)$ beam.

The $e$-like signal from $\mu^{+}$beam, $N_{e}\left(\mu^{+}\right)$, is given by the sum of $e^{+}$from the $\bar{\nu}_{\mu} \rightarrow \bar{\nu}_{e}$ appearance mode and $e^{-}$from the $\nu_{e} \rightarrow \nu_{e}$ survival mode, whereas the $\mu$-like signal, $N_{\mu}\left(\mu^{+}\right)$, is the sum of $\mu^{+}$from the $\bar{\nu}_{\mu} \rightarrow \bar{\nu}_{\mu}$ survival mode and $\mu^{-}$from the $\nu_{e} \rightarrow \nu_{\mu}$ appearance mode. The signals from the $\mu^{-}$beam, $N_{e}\left(\mu^{-}\right)$and $N_{\mu}\left(\mu^{-}\right)$, are obtained in the same way;

$$
\begin{aligned}
& N_{e}\left(\mu^{+}\right): \nu_{e} \rightarrow \nu_{e}+\bar{\nu}_{\mu} \rightarrow \bar{\nu}_{e}, \\
& N_{\mu}\left(\mu^{+}\right): \bar{\nu}_{\mu} \rightarrow \bar{\nu}_{\mu}+\nu_{e} \rightarrow \nu_{\mu}, \\
& N_{e}\left(\mu^{-}\right): \bar{\nu}_{e} \rightarrow \bar{\nu}_{e}+\nu_{\mu} \rightarrow \nu_{e} \\
& N_{\mu}\left(\mu^{-}\right): \nu_{\mu} \rightarrow \nu_{\mu}+\bar{\nu}_{e} \rightarrow \bar{\nu}_{\mu} .
\end{aligned}
$$

The signals in the $i$-th energy bin, $N_{l}^{i}\left(\mu^{+}\right)$and $N_{l}^{i}\left(\mu^{-}\right)(l=e$ or $\mu)$, are then calculated as

$$
N_{l}^{i}\left(\mu^{+}\right)=M N_{A} \int_{E_{i}}^{E_{i}+\delta E} d E_{\nu}\left\{\Phi_{\bar{\nu}_{\mu}} \cdot P_{\bar{\nu}_{\mu} \rightarrow \bar{\nu}_{l}} \cdot \sigma_{\bar{\nu}_{l}}^{\mathrm{CC}}+\Phi_{\nu_{e}} \cdot P_{\nu_{e} \rightarrow \nu_{l}} \cdot \sigma_{\nu_{l}}^{\mathrm{CC}}\right\}
$$




$$
N_{l}^{i}\left(\mu^{-}\right)=M N_{A} \int_{E_{i}}^{E_{i}+\delta E} d E_{\nu}\left\{\Phi_{\nu_{\mu}} \cdot P_{\nu_{\mu} \rightarrow \nu_{l}} \cdot \sigma_{\nu_{l}}^{\mathrm{CC}}+\Phi_{\bar{\nu}_{e}} \cdot P_{\bar{\nu}_{e} \rightarrow \bar{\nu}_{l}} \cdot \sigma_{\bar{\nu}_{l}}^{\mathrm{CC}}\right\}
$$

respectively, where $P_{\nu_{\alpha} \rightarrow \nu_{\beta}}$ and $P_{\bar{\nu}_{\alpha} \rightarrow \bar{\nu}_{\beta}}$ are the neutrino and anti-neutrino oscillation probabilities inside the earth matter, $\delta E$ is the width of the energy bin, $M$ is the mass of the detector, $N_{A}=6.017 \times 10^{23}$ is the Avogadro number, and $\sigma_{\nu_{l}}^{\mathrm{CC}}$ and $\sigma_{\bar{\nu}_{l}}^{\mathrm{CC}}$ are the $\nu_{l}$ and $\bar{\nu}_{l}$ CC cross sections of water target [24].

We show the dependences of the signals, $N_{l}^{i}\left(\mu^{+}\right)$and $N_{l}^{i}\left(\mu^{-}\right)$, on the mass hierarchy and $\sin ^{2} \theta_{\text {Aтм }}$ in Fig 2 with $10^{21}$ of unpolarized $\mu^{+}$and $\mu^{-}$decays, respectively, at $10 \mathrm{GeV}$. The left figures are the signals from the $\mu^{+}$beam and the right ones are from the $\mu^{-}$beam. The width of the bin is taken as $\delta E=1 \mathrm{GeV}$ for $E_{\nu}>2 \mathrm{GeV}$. In Fig, the signals for the normal and the inverted mass hierarchies are shown by thick and thin lines, respectively. We choose three values of $\sin ^{2} \theta_{\text {ATM }}=0.5$ (solid lines), 0.35 (dashed lines), and 0.65 (dotdashed lines), where the last two values give $\sin ^{2} 2 \theta_{\text {Атм }}=0.91$. The other parameters with the constant matter density $\rho$ are

$$
\begin{aligned}
\delta m_{\mathrm{ATM}}^{2} & =3 \times 10^{-3} \mathrm{eV}^{2}, & & \delta m_{\mathrm{SOL}}^{2}=7 \times 10^{-5} \mathrm{eV}^{2}, \\
\sin ^{2} 2 \theta_{\mathrm{SOL}} & =0.85, & & \delta_{\mathrm{MNS}}=0^{\circ}, \\
\sin ^{2} 2 \theta_{\mathrm{RCT}} & =0.06, & & \rho=3 \mathrm{~g} / \mathrm{cm}^{3} .
\end{aligned}
$$

Fig 3 shows the dependences of the expected number of events, $N_{l}^{i}\left(\mu^{+}\right)$and $N_{l}^{i}\left(\mu^{-}\right)$, on $\sin ^{2} 2 \theta_{\mathrm{RCT}}$ assuming the normal hierarchy for the same experimental setup as in Fig 2, We take three values of $\sin ^{2} 2 \theta_{\mathrm{RCT}}=0.1$ (dashed lines), 0.06 (solid lines), and 0 (dotted lines). The other parameters are taken as eq.(5) and $\sin ^{2} \theta_{\text {Атм }}=0.5$. In Fig 3 , we also show the number of background events by shaded bars, where In this study, we take account of the events coming from $\tau$ pure-leptonic-decays $N_{l}\left(\mu^{ \pm}, \tau \rightarrow l\right)$, neutral-current (NC) events, $N_{l}\left(\mu^{ \pm}, \mathrm{NC}\right)$, and $\tau$ hadronic-decays, $N_{l}\left(\mu^{ \pm}, \tau \rightarrow\right.$ had $)$. The last two processes can contribute to $e$-like events, where produced $\pi^{0}$ 's mimic the electron shower in the detector.

The contribution from the survival mode dominates each signal but that in $N_{\mu}\left(\mu^{+}\right)$and $N_{\mu}\left(\mu^{-}\right)$vanish at around $E_{\nu} \simeq 5 \mathrm{GeV}$ for $\sin ^{2} 2 \theta_{\text {Атм }}=1$ because of the nearly maximum oscillation. In $N_{e}\left(\mu^{+}\right)$and $N_{\mu}\left(\mu^{-}\right)$, the contributions from the appearance mode, $\bar{\nu}_{\mu} \rightarrow \bar{\nu}_{e}$ and $\bar{\nu}_{e} \rightarrow \bar{\nu}_{\mu}$, respectively, are suppressed for the normal hierarchy, because of the large matter effects, which suppresses $P_{\bar{\nu}_{\mu} \leftrightarrow \bar{\nu}_{e}}$ by a factor of $1 / 6$ at $E_{\nu} \sim 5 \mathrm{GeV}$ [13], and also because of small anti-neutrino CC cross sections, $\sigma_{\bar{\nu}_{l}} / \sigma_{\nu_{l}} \simeq 1 / 2$. Owing to these double suppressions of the anti-neutrino appearance contributions, $N_{e}\left(\mu^{+}\right)$measures $P_{\nu_{e} \rightarrow \nu_{e}}$ and $N_{\mu}\left(\mu^{-}\right)$measures $P_{\nu_{\mu} \rightarrow \nu_{\mu}}$ in the normal hierarchy. Because of this, $N_{e}\left(\mu^{+}\right)$is very insensitive to the parameters $\sin ^{2} \theta_{\text {ATM }}$ and $\delta_{\text {MNS }}$ that affect the transition probabilities $P_{\nu_{\mu} \leftrightarrow \nu_{e}}$ and $P_{\bar{\nu}_{\mu} \leftrightarrow \bar{\nu}_{e}}$. Insensitivity of $N_{e}\left(\mu^{+}\right)$to $\sin ^{2} \theta_{\text {ATM }}$ is shown clearly in Fig 2, The measurement of $N_{e}\left(\mu^{+}\right)$hence gives us an opportunity to measure $\sin ^{2} 2 \theta_{\mathrm{RCT}}$ uniquely in the normal 

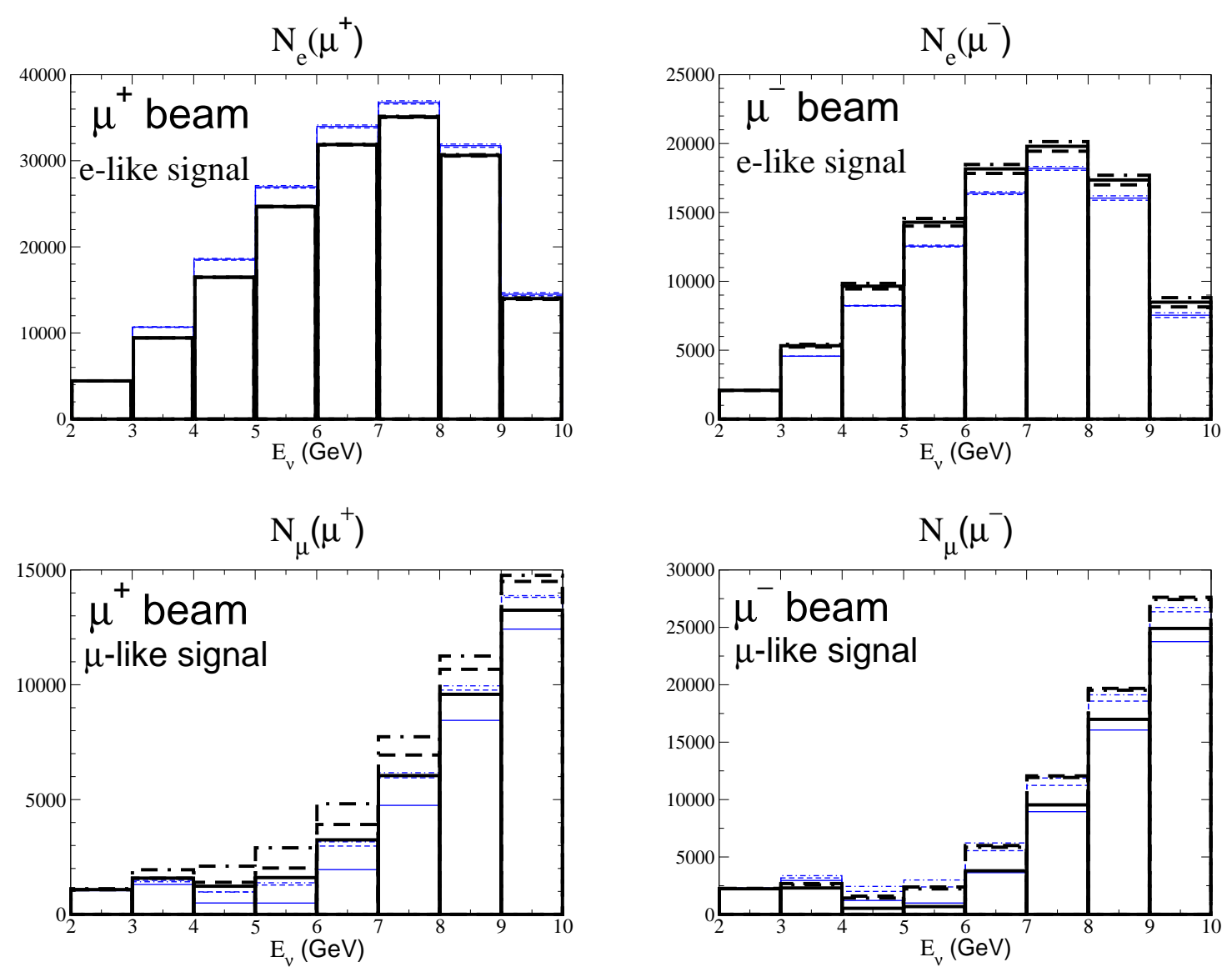

Figure 2: Number of $e$ - and $\mu$-like signals for $10^{21}$ decaying $\mu^{+}$(left) and $\mu^{-}$(right) each at $10 \mathrm{GeV}$ for the normal-hierarchy (thick lines) and the inverted hierarchy (thin lines) with three values of $\sin ^{2} \theta_{\text {ATM }}=0.5$ (solid lines), 0.35 (dashed lines), and 0.65 (dot-dashed lines). The other parameters are taken as in eq.(15).

hierarchy. The $\sin ^{2} 2 \theta_{\mathrm{RCT}}$ dependence of $N_{e}\left(\mu^{+}\right)$is clearly seen in Fig 3 .

We find, on the other hand, that the $\nu_{e}$ and $\bar{\nu}_{e}$ survival probabilities in the inverted hierarchy are always larger and smaller than those in the normal hierarchy, $P_{\nu_{e} \rightarrow \nu_{e}}^{\text {Norm }}<$ $P_{\nu_{e} \rightarrow \nu_{e}}^{\mathrm{Inv}}$ and $P_{\bar{\nu}_{e} \rightarrow \bar{\nu}_{e}}^{\mathrm{Norm}}>P_{\bar{\nu}_{e} \rightarrow \bar{\nu}_{e}}^{\mathrm{Inv}}$, because of the large matter effect. In fact they are almost independent of $\sin ^{2} 2 \theta_{\mathrm{RCT}}$ in the allowed region $0<\sin ^{2} 2 \theta_{\mathrm{RCT}} \lesssim 0.1$. Because $P_{\nu_{e} \rightarrow \nu_{e}}^{\mathrm{Norm}} \sim$ $P_{\nu_{e} \rightarrow \nu_{e}}^{\mathrm{Inv}}$ and $P_{\bar{\nu}_{e} \rightarrow \bar{\nu}_{e}}^{\mathrm{Norm}} \sim P_{\bar{\nu}_{e} \rightarrow \bar{\nu}_{e}}^{\mathrm{Inv}}$ at $\sin ^{2} 2 \theta_{\mathrm{RCT}}=0$, we find that the normal and inverted hierarchies can be distinguished by measuring $N_{e}\left(\mu^{+}\right)$and $N_{e}\left(\mu^{-}\right)$, if $\sin ^{2} 2 \theta_{\mathrm{RCT}}$ is not too small. The differences are quite significant for $\sin ^{2} 2 \theta_{\mathrm{RCT}}=0.06$, as shown in Fig 2 If the degeneracy in the mass hierarchy is lifted, the parameter $\sin ^{2} 2 \theta_{\mathrm{RCT}}$ can be measured with good accuracy by the observations of $N_{e}\left(\mu^{+}\right)$and $N_{e}\left(\mu^{-}\right)$, where the former is insensitive to $\sin ^{2} \theta_{\mathrm{ATM}}$ and $\delta_{\mathrm{MNS}}$, and so is the latter for very small $\sin ^{2} 2 \theta_{\mathrm{RCT}}$.

The $\nu_{\mu}$ survival probability $P_{\nu_{\mu} \rightarrow \nu_{\mu}}$ depends on $\sin ^{2} 2 \theta_{\text {AтM }}$ in the leading term, which is measured uniquely by $N_{\mu}\left(\mu^{-}\right)$for the normal hierarchy owing to its small dependence on 

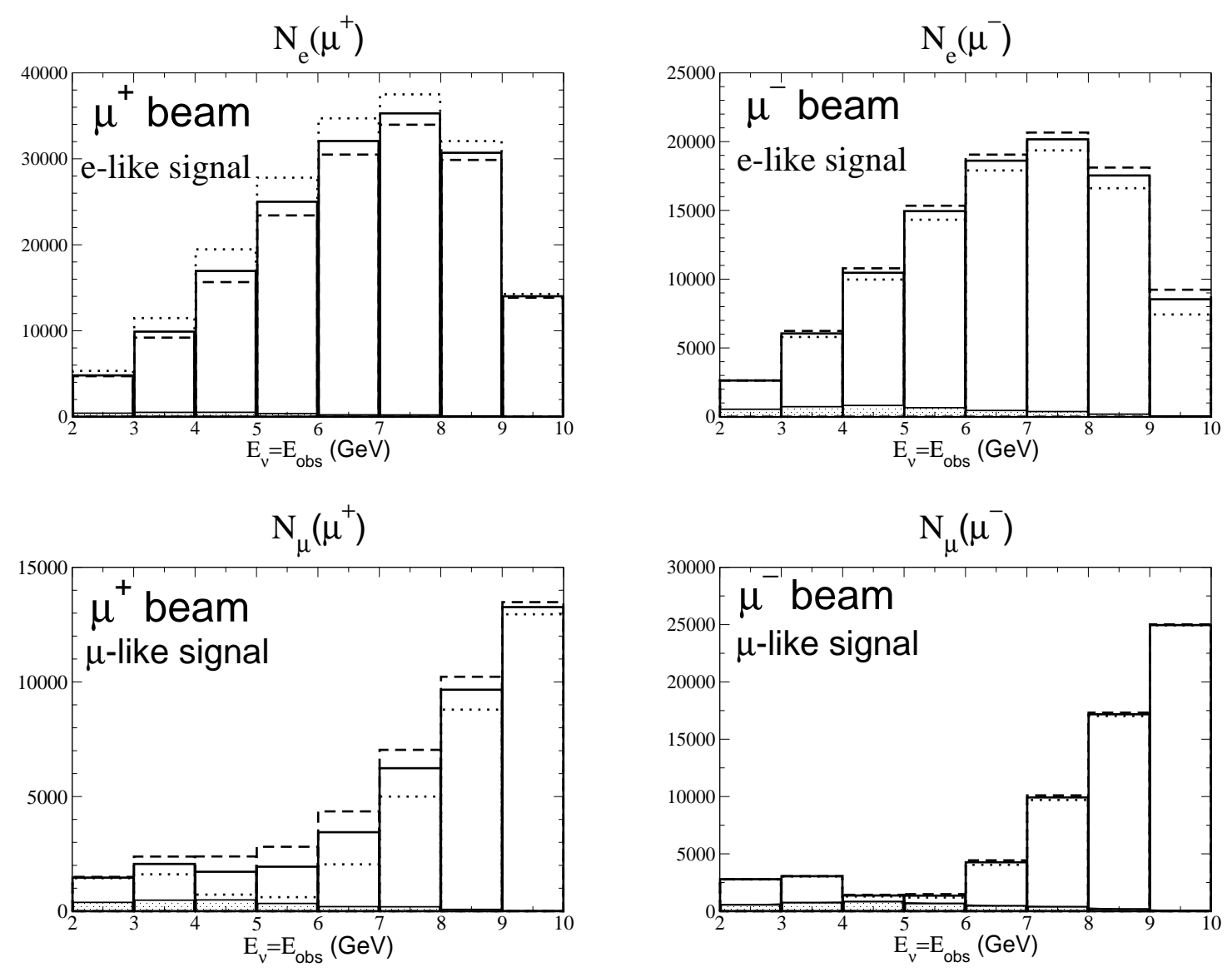

Figure 3: $\sin ^{2} 2 \theta_{\mathrm{RCT}}$ dependences of the expected number of events. The numbers are calculated for eq.(5) but for $\sin ^{2} 2 \theta_{\mathrm{RCT}}=0.1$ (dashed lines), 0.06 (solid lines), 0 (dotted lines) and $\sin ^{2} \theta_{\text {ATM }}=0.5$ with the normal-hierarchy. Shaded bars show the number of the background events. For the $\tau$ pureleptonic decay events, the horizontal scale is the observable energy after subtracting the final neutrino energies.

$\sin ^{2} 2 \theta_{\mathrm{RCT}}$ as shown in Fig 3, In Fig, the thick lines for the normal hierarchy show that the two values of $\sin ^{2} \theta_{\text {ATM }}$ that give $\sin ^{2} \theta_{\text {ATM }}=0.91$ give almost the same prediction. This is because $P_{\bar{\nu}_{e} \rightarrow \bar{\nu}_{\mu}}$ is suppressed strongly by the matter effect for the normal hierarchy. On the other hand, in case of the inverted hierarchy, we find that the degeneracy in $\sin ^{2} 2 \theta_{\text {Aтм }}$ is resolved in $N_{\mu}\left(\mu^{-}\right)$. This comes from the $\bar{\nu}_{e} \rightarrow \bar{\nu}_{\mu}$ mode which is enhanced by the matter effect and receives non-negligible contribution proportional to $\sin ^{2} \theta_{\text {ATM }} \cdot \sin ^{2} \theta_{\text {RCT }}$.

The signal of $N_{\mu}\left(\mu^{+}\right)$is the sum of the CC events from $\nu_{e} \rightarrow \nu_{\mu}$ and $\bar{\nu}_{\mu} \rightarrow \bar{\nu}_{\mu}$, which depends on $\sin ^{2} \theta_{\text {ATM }} \cdot \sin ^{2} \theta_{\text {RCT }}$ and $\sin ^{2} 2 \theta_{\text {ATM }}$, respectively. Both $\sin ^{2} \theta_{\text {ATM }}$ and $\sin ^{2} 2 \theta_{\text {ATM }}$ contributions can be seen in Fig 2, and $\sin ^{2} 2 \theta_{\mathrm{RCT}}$ dependence is clearly seen in Fig 3 , Because of the nearly maximum oscillation, the significant dependence on the sign of $\sin ^{2} \theta_{\text {ATM }}-1 / 2$ is seen at around $E_{\nu} \simeq 5 \mathrm{GeV}$.

Finally, the $N_{e}\left(\mu^{-}\right)$signal in Fig 2 shows almost linear dependence on $\sin ^{2} \theta_{\text {Aтм }}$ for the 
normal hierarchy, and that of Fig 3 shows a clear dependence on $\sin ^{2} 2 \theta_{\mathrm{RCT}}$. Those are the results of the $\nu_{\mu} \rightarrow \nu_{e}$ transition mode which is enhanced by the matter effect in the normal hierarchy.

Summing up, we come up with the following strategy for resolving the degeneracies of the three neutrino model parameters if the neutrino mass hierarchy is normal. If $\sin ^{2} 2 \theta_{\mathrm{RCT}}$ is not too small, $N_{e}\left(\mu^{+}\right)$and $N_{e}\left(\mu^{-}\right)$determine the hierarchy, and $N_{e}\left(\mu^{+}\right)$measures $\sin ^{2} 2 \theta_{\mathrm{RCT}}$. $N_{\mu}\left(\mu^{-}\right)$determines $\sin ^{2} 2 \theta_{\text {ATM }}$, and both $N_{\mu}\left(\mu^{+}\right)$and $N_{e}\left(\mu^{-}\right)$are sensitive to $\sin ^{2} \theta_{\text {ATM }}$. We can foresee the sensitivity in $\delta_{\mathrm{MNS}}$ as well, because the last two observables are sensitive to $P_{\nu_{\mu} \leftrightarrow \nu_{e}}$. The dependency in $\delta_{\mathrm{MNS}}$ and $\pi-\delta_{\mathrm{MNS}}$ may also be resolved, because their sum is sensitive to $\cos \delta_{\mathrm{MNS}}$ and their difference is sensitive to $\sin \delta_{\mathrm{MNS}}$. A more complicated strategy is needed if the neutrino mass hierarchy turned out to be inverted. We will come back to this problem elsewhere.

Bellow, we perform an exploratory study of the potential of such experiments by assuming the following simple experimental setup: A 100 kton water-Čerenkov calorimeter detector at $L=2,100 \mathrm{~km}$ away from a neutrino factory, which delivers $10^{21}$ decays of unpolarized $\mu^{+}$and $\mu^{-}$at $E_{\mu}=10 \mathrm{GeV}$.

We assume that the detector is capable of measuring the event energy, $E_{\text {obs }}=E_{\nu}($ beam $)-$ $E_{\nu}$ (final), with accuracy much better than $1 \mathrm{GeV}$ in the interval $2 \mathrm{GeV}<E_{\text {obs }}<10 \mathrm{GeV}$, and calculate the expected number of events for 8 bins with the $1 \mathrm{GeV}$ width. The probability distribution of the three neutrino parameters is then obtained from the following $\chi^{2}$ function:

$$
\begin{aligned}
\chi^{2}= & \sum_{i=1}^{8}\left\{\left(\frac{N_{e}^{i}\left(\mu^{+}\right)^{\mathrm{fit}}-N_{e}^{i}\left(\mu^{+}\right)^{\mathrm{obs}}}{\sqrt{N_{e}^{i}\left(\mu^{+}\right)^{\mathrm{obs}}}}\right)^{2}+\left(\frac{N_{\mu}^{i}\left(\mu^{+}\right)^{\mathrm{fit}}-N_{\mu}^{i}\left(\mu^{+}\right)^{\mathrm{obs}}}{\sqrt{N_{\mu}^{i}\left(\mu^{+}\right)^{\mathrm{obs}}}}\right)^{2}\right\} \\
& +\sum_{i=1}^{8}\left\{\left(\frac{N_{e}^{i}\left(\mu^{-}\right)^{\mathrm{fit}}-N_{e}^{i}\left(\mu^{-}\right)^{\mathrm{obs}}}{\sqrt{N_{e}^{i}\left(\mu^{-}\right)^{\mathrm{obs}}}}\right)^{2}+\left(\frac{N_{\mu}^{i}\left(\mu^{-}\right)^{\mathrm{fit}}-N_{\mu}^{i}\left(\mu^{-}\right)^{\mathrm{obs}}}{\sqrt{N_{\mu}^{i}\left(\mu^{-}\right)^{\mathrm{obs}}}}\right)^{2}\right\} \\
& +\left(\frac{\delta m_{\mathrm{SOL}}^{2} \text { fit }-\delta m_{\mathrm{SOL}}^{2} \text { true }}{0.1 \times \delta m_{\mathrm{SOL}}^{2 \text { true }}}\right)^{2}+\left(\frac{\sin ^{2} 2 \theta_{\mathrm{SOL}}^{\mathrm{fit}}-\sin ^{2} 2 \theta_{\mathrm{SOL}}^{\mathrm{true}}}{0.06}\right)^{2} \\
& +\left(\frac{\delta m_{\mathrm{ATM}}^{2}-\delta m_{\mathrm{ATM}}^{2 \text { true }}}{10^{-4}}\right)^{2}+\left(\frac{\sin ^{2} 2 \theta_{\mathrm{ATM}}^{\mathrm{fit}}-\sin ^{2} 2 \theta_{\mathrm{ATM}}^{\mathrm{true}}}{0.01}+\left(\frac{\rho-3.0}{0.1}\right)^{2}\right. \\
& +\sum_{\alpha=e, \mu}\left(\frac{\varepsilon_{\alpha}^{D}-1}{\Delta \varepsilon_{\alpha}^{D}}\right)^{2}+\sum_{\beta=\nu, \bar{\nu}}\left(\frac{\varepsilon_{\beta}^{\sigma}-1}{\Delta \varepsilon_{\beta}^{\sigma}}\right)^{2} \\
& +\left(\frac{\varepsilon_{\tau}-1}{\Delta \varepsilon_{\tau}}\right)^{2}+\left(\frac{\varepsilon_{e / \mathrm{NC}}-1}{\Delta \varepsilon_{e / \mathrm{NC}}}\right)^{2} .
\end{aligned}
$$


Here $N_{l}^{i}\left(\mu^{ \pm}\right)^{\text {obs }}$ are calculated as

$$
\begin{aligned}
& N_{e}^{i}\left(\mu^{ \pm}\right)^{\mathrm{obs}}=N_{e}^{i}\left(\mu^{ \pm}\right)+N_{e}^{i}\left(\mu^{ \pm}, \tau \rightarrow e\right)+N_{e}^{i}\left(\mu^{ \pm}, \tau \rightarrow \operatorname{had}\right)+N_{e}^{i}\left(\mu^{ \pm}, \mathrm{NC}\right), \\
& N_{\mu}^{i}\left(\mu^{ \pm}\right)^{\mathrm{obs}}=N_{e}^{i}\left(\mu^{ \pm}\right)+N_{\mu}^{i}\left(\mu^{ \pm}, \tau \rightarrow \mu\right),
\end{aligned}
$$

including contributions for the pure-leptonic $\tau$ decays, and the fake contributions to $N_{e}^{i}\left(\mu^{ \pm}\right)$ where the $\mathrm{NC}$ events or the $\nu_{\tau} \mathrm{CC}$ events with hadronic $\tau$ decays are mistaken as $e^{ \pm}$events. These background contributions are calculated as

$$
\begin{aligned}
& N_{l}^{i}\left(\mu^{+}, \tau \rightarrow l\right)= M N_{A} \int_{E_{i}}^{E_{i}+\delta E} d E_{\mathrm{obs}} \int_{E_{0}}^{E_{\max }} d E_{\nu} \\
&\left\{\Phi_{\bar{\nu}_{\mu}} \cdot P_{\bar{\nu}_{\mu} \rightarrow \bar{\nu}_{\tau}} \cdot \frac{d \sigma_{\bar{\nu}_{\tau}}^{\mathrm{CC}}}{d E_{\mathrm{obs}}}+\Phi_{\nu_{e}} \cdot P_{\nu_{e} \rightarrow \nu_{\tau}} \cdot \frac{d \sigma_{\nu_{\tau}}^{\mathrm{CC}}}{d E_{\mathrm{obs}}}\right\} \cdot \mathrm{B}(\tau \rightarrow l), \\
& N_{l}^{i}\left(\mu^{+}, \tau \rightarrow \mathrm{had}\right)= M N_{A} \int_{E_{i}}^{E_{i}+\delta E} d E_{\mathrm{obs}} \int_{E_{0}}^{E_{\max }} d E_{\nu} \\
&\left\{\Phi_{\bar{\nu}_{\mu}} \cdot P_{\bar{\nu}_{\mu} \rightarrow \bar{\nu}_{\tau}} \cdot \frac{d \sigma_{\bar{\nu}_{\tau}}^{\mathrm{CC}}}{d E_{\mathrm{obs}}}+\Phi_{\nu_{e}} \cdot P_{\nu_{e} \rightarrow \nu_{\tau}} \cdot \frac{d \sigma_{\nu_{\tau}}^{\mathrm{CC}}}{d E_{\mathrm{obs}}}\right\} \cdot \mathrm{B}(\tau \rightarrow \mathrm{had}) \cdot P_{e / \mathrm{NC}}, \\
& N_{e}^{i}\left(\mu^{+}, \mathrm{NC}\right)= M N_{A} \int_{E_{i}}^{E_{i}+\delta E} d E_{\nu}\left\{\Phi_{\bar{\nu}_{\mu}} \cdot \sigma_{\bar{\nu}}^{\mathrm{NC}}+\Phi_{\nu_{e}} \cdot \sigma_{\nu}^{\mathrm{NC}}\right\} \cdot P_{e / \mathrm{NC}}
\end{aligned}
$$

and likewise for the events from decaying $\mu^{-}$. Here $E_{\text {obs }}$ is calculated by subtracting the energies carried away by neutrinos in $\tau$ decays. The oscillation probabilities are calculated for a set of parameters of the three neutrino model labeled as 'true', in the normal hierarchy for a constant matter density of $\rho=3 \mathrm{~g} / \mathrm{cm}^{3}$. The statistical errors of the number of events in each bin are simply the square root of the observed number calculated as above.

In the calculation of the model predictions for these numbers, labeled as $N_{l}\left(\mu^{ \pm}\right)^{\mathrm{fit}}$, we allow all the 6 neutrino model parameters and the matter density $\rho$ to vary freely under the constraints given in the third and the fourth lines of eq.(6). For $\delta m_{\mathrm{SOL}}^{2}$ and $\sin ^{2} 2 \theta_{\mathrm{SOL}}$, we set the $1 \sigma$ errors which will be achieved by the KamLAND experiment $[8]$. The LBL neutrino oscillation experiment Tokai-to-SK will determine $\delta m_{\text {ATM }}^{2}$ and $\sin ^{2} 2 \theta_{\text {ATM }}$ with the accuracy $\delta\left(\delta m_{\text {ATM }}^{2}\right) \lesssim 10^{-4}$ and $\delta\left(\sin ^{2} 2 \theta_{\text {ATM }}\right)<0.01$, respectively [15]. We assume that the average matter density along the baseline will be known with $0.1 \mathrm{~g} / \mathrm{cm}^{3}$ accuracy.

Furthermore, the following systematic effects are taken into account in our fit. Although we can probably neglect the flux uncertainties in the neutrino factory experiments, the observed numbers of events should depend on the detection efficiencies, and the expected numbers of events suffer from the uncertainties in the neutrino $\mathrm{CC}$ cross sections. We introduce the detection efficiency parameter $\varepsilon_{e}^{D}$ and $\varepsilon_{\mu}^{D}$ separately for ' $e$ '-like and ' $\mu$ '-like events, respectively. For simplicity, we set $\varepsilon_{e}^{D}=\varepsilon_{\mu}^{D}=1$ in calculating the expected number of events, but allow for their errors

$$
\varepsilon_{e}^{D}=1 \pm \Delta \varepsilon_{e}^{D}, \quad \varepsilon_{\mu}^{D}=1 \pm \Delta \varepsilon_{\mu}^{D} .
$$


Here we assume that the fiducial volume uncertainty can be accounted for as a part of the efficiency uncertainties. For the cross sections, we assume that the ratio $\sigma_{\nu_{\mu}}^{\mathrm{CC}} / \sigma_{\nu_{e}}^{\mathrm{CC}}$ and $\sigma_{\bar{\nu}_{\mu}}^{\mathrm{CC}} / \sigma_{\bar{\nu}_{e}}^{\mathrm{CC}}$ will be determined accurately in the future and introduce the following uncertainty factors

$$
\frac{\sigma_{\nu_{\mu}}^{\mathrm{CC}}}{\left(\sigma_{\nu_{\mu}}^{\mathrm{CC}}\right)_{\text {input }}}=\frac{\sigma_{\nu_{e}}^{\mathrm{CC}}}{\left(\sigma_{\nu_{e}}^{\mathrm{CC}}\right)_{\text {input }}}=\varepsilon_{\nu}^{\sigma}=1 \pm \Delta \varepsilon_{\nu}^{\sigma}, \quad \frac{\sigma_{\bar{\nu}_{\mu}}^{\mathrm{CC}}}{\left(\sigma_{\bar{\nu}_{\mu}}^{\mathrm{CC}}\right)_{\text {input }}}=\frac{\sigma_{\bar{\nu}_{e}}^{\mathrm{CC}}}{\left(\sigma_{\bar{\nu}_{e}}^{\mathrm{CC}}\right)_{\text {input }}}=\varepsilon_{\bar{\nu}}^{\sigma}=1 \pm \Delta \varepsilon_{\bar{\nu}}^{\sigma}
$$

These systematic uncertainties eq.(9) and eq.(10) play important roles in determining the ultimate accuracy of the experiments when the statistical errors get smaller, and appear in the fifth line of eq.(6). Below, we show our results for $\Delta \varepsilon_{e}^{D}=\Delta \varepsilon_{\mu}^{D}=\Delta \varepsilon_{\nu}^{\sigma}=\Delta \varepsilon_{\bar{\nu}}^{\sigma}=0$ and $2 \%$, so that the impacts of reducing these systematic uncertainties can be inferred.

We also take account of the systematic errors for the uncertainty in the branching fractions of $\tau$ leptonic-decays as

$$
\frac{B\left(\tau \rightarrow e \bar{\nu}_{e} \nu_{\tau}\right)}{B\left(\tau \rightarrow e \bar{\nu}_{e} \nu_{\tau}\right)_{\text {input }}}=\frac{B\left(\tau \rightarrow \mu \bar{\nu}_{\mu} \nu_{\tau}\right)}{B\left(\tau \rightarrow \mu \bar{\nu}_{\mu} \nu_{\tau}\right)_{\text {input }}}=\varepsilon_{\tau}=1 \pm \Delta \varepsilon_{\tau}
$$

with $B\left(\tau \rightarrow e \bar{\nu}_{e} \nu_{\tau}\right)_{\text {input }}=0.178$ and $B\left(\tau \rightarrow \mu \bar{\nu}_{\mu} \nu_{\tau}\right)_{\text {input }}=0.174$ [26], and $\Delta \varepsilon_{\tau}=0.1$. For the $\mathrm{NC}$ events, we set the mean value of the $e / \mathrm{NC}$ misidentification probability as $\left(P_{e / \mathrm{NC}}\right)_{\text {input }}=0.25 \%$ by using the estimations from the $\mathrm{K} 2 \mathrm{~K}$ experiment [2]. The uncertainty factors are then introduced as

$$
\frac{P_{e / \mathrm{NC}}}{\left(P_{e / \mathrm{NC}}\right)_{\text {input }}}=\varepsilon_{e / \mathrm{NC}}=1 \pm \Delta \varepsilon_{e / \mathrm{NC}}
$$

with $\Delta \varepsilon_{e / \mathrm{NC}}=0.1$. Contributions from these systematics are accounted for as the last two terms of eq.(6).

We show in Fig $4 \chi_{\min }^{2}$ as a function of $\sin ^{2} 2 \theta_{\mathrm{RCT}}^{\text {fit }}$ when the event numbers calculated for the normal hierarchy are analyzed by assuming the inverted hierarchy. The results shown in Fig 4 (a) are obtained when the detecting efficiencies and the cross sections are known exactly, $\Delta \varepsilon_{e}^{D}=\Delta \varepsilon_{\mu}^{D}=\Delta \varepsilon_{\nu}^{\sigma}=\Delta \varepsilon_{\nu}^{\sigma}=0$. Because of $P_{\nu_{e} \rightarrow \nu_{e}}^{\mathrm{Norm}}<P_{\nu_{e} \rightarrow \nu_{e}}^{\mathrm{Inv}}$ and $P_{\bar{\nu}_{e} \rightarrow \bar{\nu}_{e}}^{\mathrm{Norm}}>P_{\bar{\nu}_{e} \rightarrow \bar{\nu}_{e}}^{\mathrm{Inv}}$, the normal and inverted hierarchies can be distinguished by measuring $N_{e}\left(\mu^{+}\right)$and $N_{e}\left(\mu^{-}\right)$. Normal hierarchy can be established at $5 \sigma$ level when $\sin ^{2} 2 \theta_{\text {RCT }}^{\text {true }} \gtrsim 0.01$, and at $3 \sigma$ level when $\sin ^{2} 2 \theta_{\mathrm{RCT}}^{\text {true }} \gtrsim 0.004$. However, the differences of the $e$-like signals between the normal and the inverted hierarchy is reduced to $\sim 1 \%$ when $\sin ^{2} 2 \theta_{\mathrm{RCT}}=0.004$. It is hence necessary to measure the cross section of $\nu_{e}$ and $\bar{\nu}_{e}$ CC events with an accuracy better than $1 \%$. In Fig 4 (b), we show the results when we account for $2 \%$ uncertainties in the cross sections and the efficiencies, $\Delta \varepsilon_{e}^{D}=\Delta \varepsilon_{\mu}^{D}=\Delta \varepsilon_{\nu}^{\sigma}=\Delta \varepsilon_{\bar{\nu}}^{\sigma}=0.02$. The hierarchy discrimination power of the experiment is reduced significantly, when the 5(3) $\sigma$ discrimination is now possible only when $\sin ^{2} 2 \theta_{\text {RCT }}^{\text {true }} \gtrsim 0.1(0.008)$. 
(a)

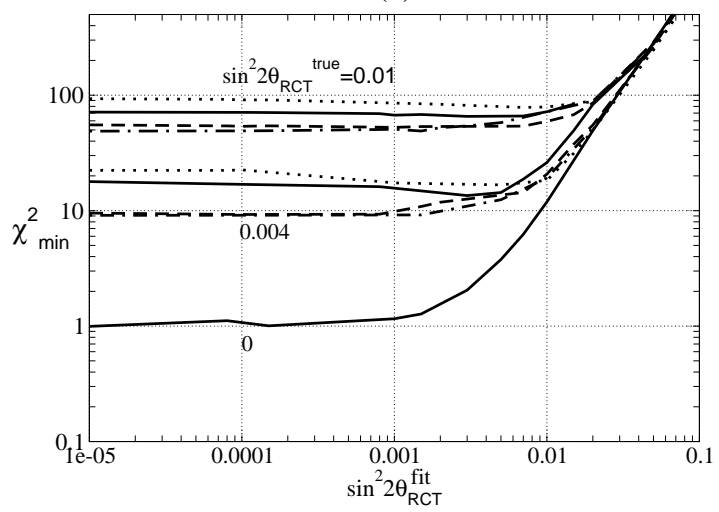

(b)

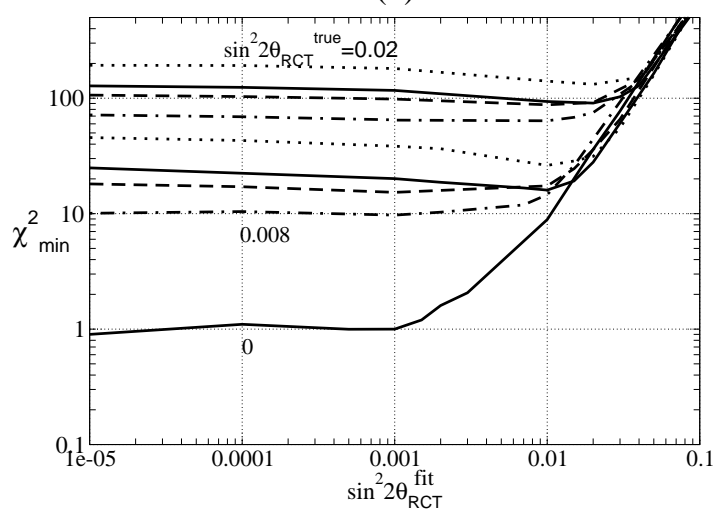

Figure 4: $\chi_{\min }^{2}$ as a function of the fitting parameter of $\sin ^{2} 2 \theta_{\mathrm{RCT}}^{\mathrm{fit}}$ by assuming the inverted hierarchy. The resluts are for $10^{21} \mu^{+}$and $\mu^{-}$decays for a 100 kton water target at $L=2,100 \mathrm{~km}$. The input data are calculated for the 'true' values in eq. (13) by assuming the normal hierarchy The 12 sets of the input data are labeled by $\sin ^{2} 2 \theta_{\mathrm{RCT}}^{\text {true }}=0.01,0.004$ and 0 for (a), $0.02,0.008$ and 0 for (b), with $\delta_{\text {true }}^{\text {tos }}=0^{\circ}$ (solid lines), $90^{\circ}$ (dotted lines), $180^{\circ}$ (dashed lines), $270^{\circ}$ (dot-dashed lines). $\Delta \varepsilon_{e}^{D}=\Delta \varepsilon_{\mu}^{D}=\Delta \varepsilon_{\nu}^{\sigma}=\Delta \varepsilon_{\bar{\nu}}^{\sigma}=0$ for (a), and $\Delta \varepsilon_{e}^{D}=\Delta \varepsilon_{\mu}^{D}=\Delta \varepsilon_{\nu}^{\sigma}=\Delta \varepsilon_{\bar{\nu}}^{\sigma}=0.02$ for (b).

The input data for Fig 4 (a) and Fig 4 (b) are generated by assuming the normal hierarchy with the following 'true' values :

$$
\begin{array}{rlrl}
\sin ^{2} \theta_{\mathrm{ATM}}^{\text {true }} & =0.5, & \delta m_{\mathrm{ATM}}^{2 \text { true }}=3 \times 10^{-3} \mathrm{eV}^{2}, \\
\sin ^{2} 2 \theta_{\mathrm{SOL}}^{\text {true }} & =0.85, & \delta m_{\mathrm{SOL}}^{2 \text { true }}=7 \times 10^{-5} \mathrm{eV}^{2}, \\
\sin ^{2} 2 \theta_{\mathrm{RCT}}^{\text {true }} & =0.01,0.004,0(\text { for }(\mathrm{a})), 0.02,0.008,0(\text { for }(\mathrm{b})), \\
\delta_{\mathrm{MNS}}^{\text {true }} & =0^{\circ}, 90^{\circ}, 180^{\circ}, 270^{\circ} .
\end{array}
$$

The 12 sets of the input data are labeled as $\sin ^{2} 2 \theta_{\mathrm{RCT}}^{\text {true }}=0.01,0.004$ and 0 in Fig 4 (a) and $0.02,0.008$ and 0 in Fig 4 (b) with $\delta_{\text {MNS }}^{\text {true }}=0^{\circ}$ (solid lines), 90 (dotted lines), $180^{\circ}$ (dashed lines), $270^{\circ}$ (dot-dashed lines). We then fit the data by assuming the inverted hierarchy by allowing all the parameters to vary freely, and obtain the $\chi_{\min }^{2}$ values plotted in the figures.

We show in Fig $\chi_{\text {min }}^{2}$ as a function of $\sin ^{2} \theta_{\mathrm{ATM}}^{\mathrm{fit}}$ for (a) $\Delta \varepsilon_{e}^{D}=\Delta \varepsilon_{\mu}^{D}=\Delta \varepsilon_{\nu}^{\sigma}=\Delta \varepsilon_{\nu}^{\sigma}=0$, and (b) $\Delta \varepsilon_{e}^{D}=\Delta \varepsilon_{\mu}^{D}=\Delta \varepsilon_{\nu}^{\sigma}=\Delta \varepsilon_{\bar{\nu}}^{\sigma}=0.02$. Input data are calculated for $\sin ^{2} \theta_{\text {ATM }}^{\text {true }}=$ $0.35,0.4$, and $0.45\left(\sin ^{2} 2 \theta_{\text {ATM }}^{\text {true }}=0.91,0.96\right.$, and 0.99 , respectively) with three values of $\sin ^{2} 2 \theta_{\mathrm{RCT}}^{\text {true }}=0.06$ (solid lines), $0.02[0.08]$ (dotted lines) and 0.01 [0.1] (dashed lines) for (a) $[(\mathrm{b})]$ and $\delta_{\text {MNS }}^{\text {true }}=0^{\circ}$ with the normal hierarchy. The values of the other parameters are taken as in eq.(13). The $\chi_{\min }^{2}$ function is found by varying the fitting parameters within the normal hierarchy. We see that each $\chi_{\min }^{2}$ has two dips at the value of $\sin ^{2} \theta_{\text {ATM }}^{\text {true }}$ which give the same $\sin ^{2} 2 \theta_{\text {ATM }}$. The results in Fig 5 (a) show that we can resolve the degeneracy 
(a)

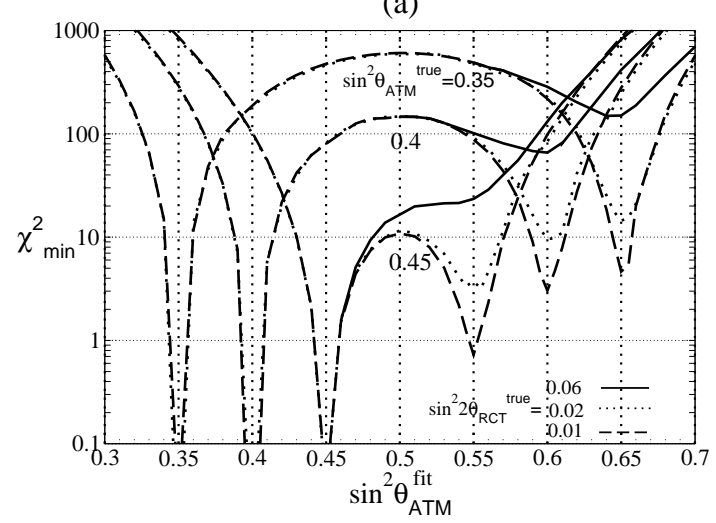

(b)

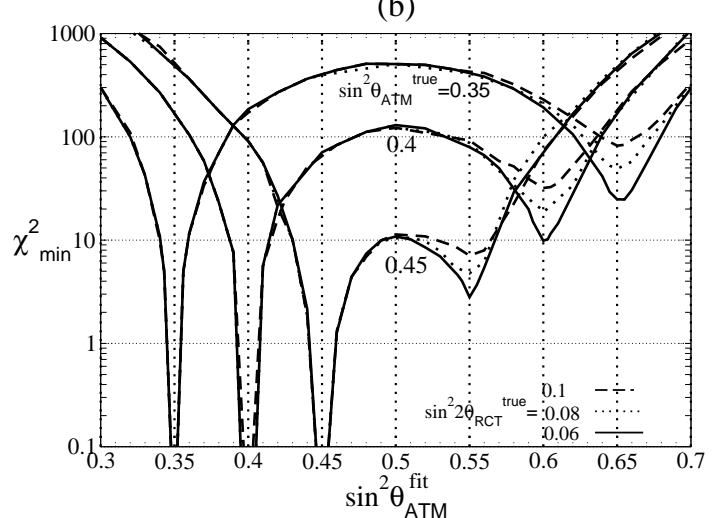

Figure 5: $\chi_{\min }^{2}$ as a function of the fitting parameters $\sin ^{2} \theta_{\mathrm{ATM}}^{\mathrm{fit}}$ for (a) $\Delta \varepsilon_{e}^{D}=\Delta \varepsilon_{\mu}^{D}=$ $\Delta \varepsilon_{\nu}^{\sigma}=\Delta \varepsilon_{\bar{\nu}}^{\sigma}=0$ and (b) $\Delta \varepsilon_{e}^{D}=\Delta \varepsilon_{\mu}^{D}=\Delta \varepsilon_{\nu}^{\sigma}=\Delta \varepsilon_{\bar{\nu}}^{\sigma}=0.02$. The input data of the events are calculated for $\sin ^{2} \theta_{\mathrm{ATM}}^{\text {true }}=0.35,0.4$, and 0.45 with $\sin ^{2} 2 \theta_{\mathrm{RCT}}^{\text {true }}=0.06$ (solid lines), 0.02 [0.08] (dottted lines) and $0.01[0.1]$ (dashed lines) for (a) [(b)] and $\delta_{\mathrm{MNS}}^{\text {true }}=0^{\circ}$. The other input values are the same as in eq.(13).

at $3 \sigma$ level when $\sin ^{2} \theta_{\mathrm{ATM}}^{\text {true }}=0.4(0.45)$ for $\sin ^{2} 2 \theta_{\mathrm{RCT}}^{\text {true }} \gtrsim 0.02(0.04)$. The degeneracy in $\sin ^{2} \theta_{\text {ATM }}$ can be resolved by the $\nu_{e} \rightarrow \nu_{\mu}$ mode in $N_{\mu}\left(\mu^{+}\right)$and $\nu_{\mu} \rightarrow \nu_{e}$ mode in $N_{e}\left(\mu^{-}\right)$ whose leading terms are proportional to $\sin ^{2} \theta_{\text {ATM }} \sin ^{2} \theta_{\text {RCT }}$, when $\sin ^{2} 2 \theta_{\text {RCT }}$ and $\sin ^{2} 2 \theta_{\text {ATM }}$ are determined by the survival mode in $N_{e}\left(\mu^{+}\right)$and $N_{\mu}\left(\mu^{-}\right)$, respectively. In Fig 5 (b) where we take account of $2 \%$ systematic errors in the CC cross sections and the detection efficiencies, the degeneracy in the sign of $\sin ^{2} \theta_{\text {ATM }}-1 / 2$ can be resolved at $3 \sigma$ level when $\sin ^{2} \theta_{\mathrm{ATM}}^{\text {true }}=0.4$ for $\sin ^{2} 2 \theta_{\mathrm{RCT}}^{\text {true }} \gtrsim 0.06$. In the case of $\sin ^{2} \theta_{\mathrm{ATM}}^{\text {true }}=0.45$, the determination of the sign is possible at $3 \sigma$ level only when $\sin ^{2} 2 \theta_{\mathrm{RCT}}^{\text {true }} \sim 0.1$.

In Fig [6 we show allowed regions in the plane of $\sin ^{2} 2 \theta_{\mathrm{RCT}}^{\mathrm{fit}}$ and $\delta_{\mathrm{MNS}}^{\mathrm{fit}}$ when $\sin ^{2} 2 \theta_{\mathrm{RCT}}^{\text {true }}=$ $0.02,0.06$, and 0.1 with $\delta_{\text {MNS }}^{\text {true }}=0^{\circ}$ (upper left), $90^{\circ}$ (upper right), $180^{\circ}$ (lower left), and $270^{\circ}$ (lower right) in the normal hierarchy and the other parameters are eq.(13a) and eq.(13b). The errors in the efficiencies and the cross section are set as $\Delta \varepsilon_{e}^{D}=\Delta \varepsilon_{\mu}^{D}=\Delta \varepsilon_{\nu}^{\sigma}=\Delta \varepsilon_{\bar{\nu}}^{\sigma}=$ 0.02. In each figure, the input parameter points are shown by solid-circles. The normal hierarchy is assumed in the fitting. The regions where $\chi_{\min }^{2}<1,4$, and 9 are depicted by solid, dashed, and dotted boundaries, respectively.

The figures for $\delta_{\text {MNS }}^{\text {true }}=90^{\circ}$ and $\delta_{\text {MNS }}^{\text {true }}=270^{\circ}$ show that the CP phase $\delta_{\text {MNS }}$ can be constrained locally around the 'true' points. We can discriminate the maximal $\mathrm{CP}$ violation cases $\delta_{\mathrm{MNS}}^{\text {true }}=90^{\circ}, 270^{\circ}$ from the $\mathrm{CP}$ conserving cases $\delta_{\mathrm{MNS}}=0^{\circ}, 180^{\circ}$ at $3 \sigma$ level for $\sin ^{2} 2 \theta_{\mathrm{RCT}}^{\text {true }} \gtrsim 0.02$. The sensitivity is based essentially on the $\mathrm{T}$ violating difference between $P_{\nu_{e} \rightarrow \nu_{\mu}}$ and $P_{\nu_{\mu} \rightarrow \nu_{e}}$. Because the matter effects enhance both of these transitions whereas suppressed strongly the transitions $\bar{\nu}_{\mu} \rightarrow \bar{\nu}_{e}$ and $\bar{\nu}_{e} \rightarrow \bar{\nu}_{\mu}, N_{\mu}\left(\mu^{+}\right)$is sensitive to $P_{\nu_{e} \rightarrow \nu_{\mu}}$ 

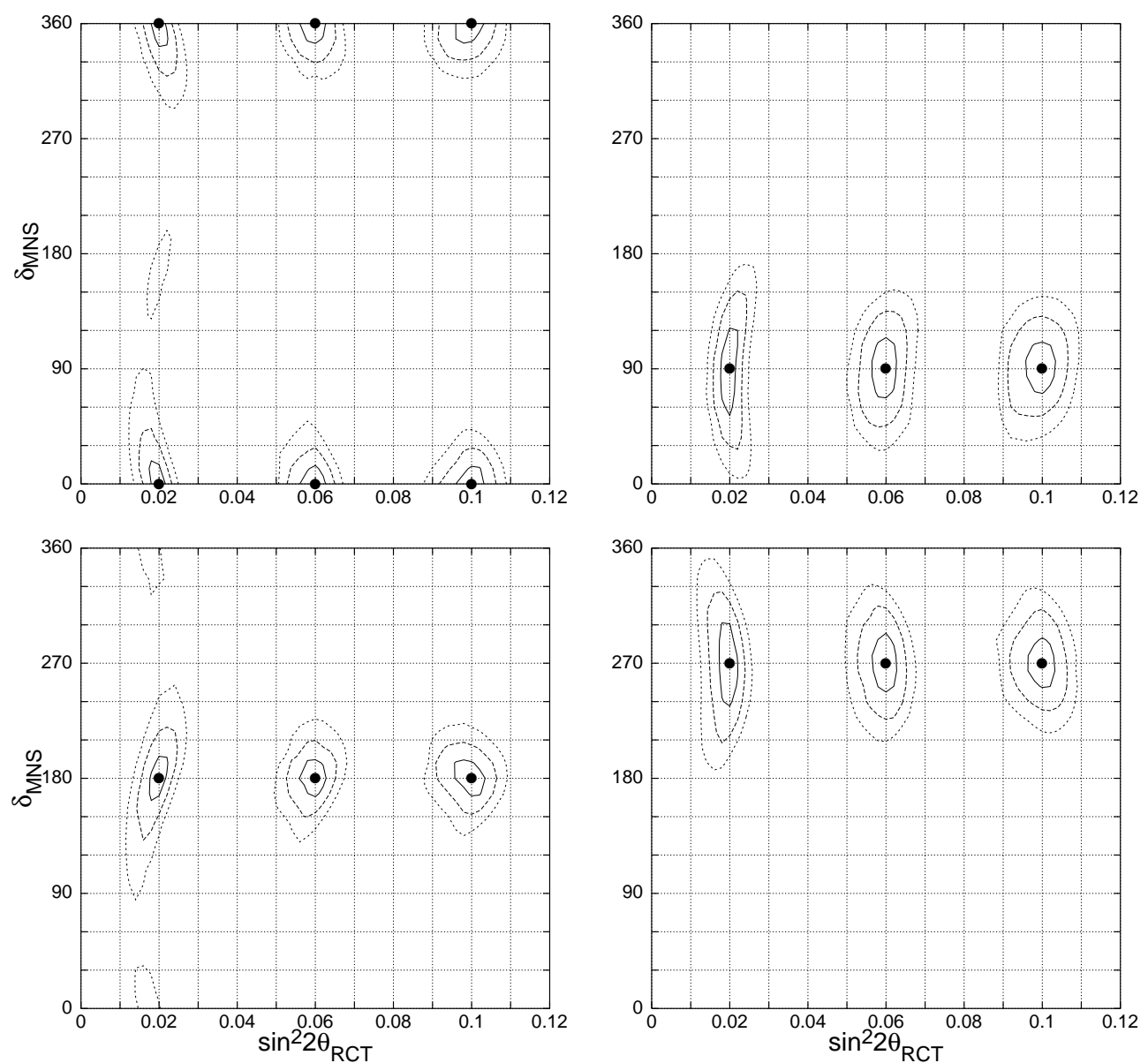

Figure 6: Allowed regions in the plane of $\sin ^{2} 2 \theta_{\mathrm{RCT}}$ and $\delta_{\mathrm{MNS}}$ for $\sin ^{2} 2 \theta_{\mathrm{RCT}}^{\text {true }}=0.02,0.06$, and 0.1 with $\delta_{\text {MNS }}^{\text {true }}=0^{\circ}$ (upper left), $90^{\circ}$ (upper right), $180^{\circ}$ (lower left), and $270^{\circ}$ (lower right). The errors in the efficiencies and the cross section are taken to $\Delta \varepsilon_{e}^{D}=\Delta \varepsilon_{\mu}^{D}=$ $\Delta \varepsilon_{\nu}^{\sigma}=\Delta \varepsilon_{\bar{\nu}}^{\sigma}=0.02$. In each figure, the input parameter points are shown by solid-circles. The regions where $\chi_{\min }^{2}<1,4$, and 9 are depicted by solid, dashed, and dotted boundaries, respectively.

and $N_{e}\left(\mu^{-}\right)$is sensitive to $P_{\nu_{\mu} \rightarrow \nu_{e}}$.

For $\delta_{\text {MNS }}^{\text {true }}=0^{\circ}$ and $\delta_{\text {MNS }}^{\text {true }}=180^{\circ}$, we can constrain $\delta_{\text {MNS }}$ within $\pm 45^{\circ}$ accuracy for $\sin ^{2} 2 \theta_{\mathrm{RCT}}^{\text {true }}=0.06$ and 0.1 , while the fake solutions appear for $\sin ^{2} 2 \theta_{\mathrm{RCT}}^{\text {true }}=0.02$ at $3 \sigma$ level. This is essentially because both $\nu_{e} \rightarrow \nu_{\mu}$ and $\nu_{\mu} \rightarrow \nu_{e}$ transitions have terms proportional to $\sin ^{2} 2 \theta_{\mathrm{RCT}} \cos \delta_{\mathrm{MNS}} ; N_{e}\left(\mu^{+}\right)$measures $\sin ^{2} 2 \theta_{\mathrm{RCT}}$ uniquely, and the sum of $N_{\mu}\left(\mu^{+}\right)$and $N_{e}\left(\mu^{-}\right)$is sensitive to $\sin ^{2} 2 \theta_{\mathrm{RCT}} \cos \delta_{\mathrm{MNS}}$ while the difference is sensitive to $\sin ^{2} 2 \theta_{\mathrm{RCT}} \sin \delta_{\mathrm{MNS}}$. In our previous study of J-PARC superbeam and Hyper-Kamiokande [16], it was difficult to resolve the degeneracy which gives same values for $\sin ^{2} 2 \theta_{\mathrm{RCT}} \cos \delta_{\mathrm{MNS}}$.

In this letter, we examine the capability of the VLBL neutrino experiment with a neutrino factory at J-PARC in Tokai Village to resolve degeneracies in the neutrino oscillation parameters. We find that a large segmented water-Čerenkov calorimeter detector placed 
at a few thousand $\mathrm{km}$ away, such as a proposed BAND detector [21] in Beijing, can be very effective in solving the problem. The charge identification capability at the detector is not required. This is mainly because the $e^{ \pm}$events from the $\mu^{+}$beam measure the $\nu_{e} \rightarrow \nu_{e}$ survival rate rather accurately while the $e^{ \pm}$events from the $\mu^{-}$beam are more sensitive to the $\nu_{\mu} \rightarrow \nu_{e}$ transition rate, for the normal hierarchy, where the earth matter effects strongly suppress the $\bar{\nu}_{\mu} \rightarrow \bar{\nu}_{e}$ and $\bar{\nu}_{e} \rightarrow \bar{\nu}_{\mu}$ transitions. On the other hand, if the hierarchy is inverted, the $\nu_{\mu} \rightarrow \nu_{e}$ and $\nu_{e} \rightarrow \nu_{\mu}$ transitions are suppressed and the $e^{ \pm}$events from $\mu^{+}$beam are sensitive to the $\bar{\nu}_{\mu} \rightarrow \bar{\nu}_{e}$ transitions and those from $\mu^{-}$beam are sensitive to $\bar{\nu}_{e} \rightarrow \bar{\nu}_{e}$. Because of the strong matter effects in the $\nu_{\mu} \leftrightarrow \nu_{e}$ and $\bar{\nu}_{\mu} \leftrightarrow \bar{\nu}_{e}$ transitions processes, we can resolve not only the neutrino mass hierarchy but also the degeneracies in the sign of $\sin ^{2} \theta_{\mathrm{ATM}}-1 / 2$ and those between $\delta_{\mathrm{MNS}}$ and $180^{\circ}-\delta_{\mathrm{MNS}}$.

The following numerical results are found at $3 \sigma$ level for $10^{21}$ decaying unpolarized $\mu^{+}$and $\mu^{-}$at $10 \mathrm{GeV}$ when we take into account $2 \%$ uncertainties in the detection efficiencies and the $\nu_{e}$ and $\nu_{\mu} \mathrm{CC}$ cross section measurements and assume that the energy resolution is significantly smaller than $1 \mathrm{GeV}$. The mass hierarchy can be determined for $\sin ^{2} 2 \theta_{\mathrm{RCT}} \gtrsim 0.008$, which is remarkably smaller than the corresponding limit in the VLBL experiment with the conventional beams from J-PARC [13. When $\sin ^{2} 2 \theta_{\text {RCT }} \gtrsim 0.06$, the degeneracy in the sign of $\sin ^{2} \theta_{\text {ATM }}-1 / 2$ can be lifted for $\sin ^{2} 2 \theta_{\text {ATM }}=0.96$. The CP violating phase $\delta_{\mathrm{MNS}}$ can be uniquely constrained for $\sin ^{2} 2 \theta_{\mathrm{RCT}} \gtrsim 0.02$ if its true value is around $90^{\circ}$ or $270^{\circ}$, while if it is around $0^{\circ}$ or $180^{\circ}$, the mirror solution at $180^{\circ}-\delta_{\mathrm{MNS}}$ can be excluded for $\sin ^{2} 2 \theta_{\mathrm{RCT}} \gtrsim 0.03$. It is essential to have both $\mu^{+}$and $\mu^{-}$beams to obtain the above results, but it is not necessary for the detector to have the charge identification capability.

\section{Acknowledgments}

The authors wish to thank stimulating discussions with Y. Hayato, T. Kobayashi, M. Koike, J. Sato, Y. F. Wang and I. Watanabe. They would also like to thank the refree whose important comments on the treatments of the systematic errors made our exploratory study more realistic. The work of MA is supported in part by the Academy of Finland and the Japan Society for the Promotion of Science (JSPS). The work of KH has been partially supported by Japan-China Scientific Cooperation Program of JSPS. The work of NO is supported in part by a grand from the US Department of Energy, DE-FG05-92ER40709.

\section{References}

[1] Y. Itow, presented at ICFP2003, KIAS, Oct. 2003.

[2] K. Nishikawa, presented at ICFP2003, KIAS, Oct. 2003; K. Nishikawa, presented at lepton photon 2003, Fermilab, Aug. 2003. 
[3] L. Wolfenstein, Phys. Rev. D17, 2369 (1978).

[4] S.P. Mikheyev and A.Yu. Smirnov, Yad. Fiz. 42, 1441 (1985), [Sov.J.Nucl.Phys.42, 913 (1986)]; Nuovo Cimento C9, 17 (1986).

[5] B.T. Cleveland et al., Astrophys. J., 496505 (1998); J.N. Abdurashitov et al., J.Exp.Theor.Phys., 95 181, (2002) ; W. Hampel et al., Phys. Lett. B447, 127 (1999); M. Altmann et al., Phys. Lett. B490, 16 (2000).

[6] Y. Koshio, (Super-Kamiokande Collaboration) hep-ex/0306002 Super-Kamiokande Collaboration, Phys. Rev. D69, 011104 (2004).

[7] S.N. Ahmed et al. (SNO collaboration), Phys. Rev. Lett. 92, 181301 (2004).

[8] K. Eguchi et al. (KamLAND Collaboration), Phys. Rev. Lett. 90, 021802 (2003).

[9] M. Apollonio et al. (CHOOZ Collaboration), Eur. Phys. J. C27, 331 (2003).

[10] F. Boehm, et al., Phys. Rev. Lett. 84, 3764 (2000); Phys. Rev. D62, 072002 (2000); Phys. Rev. D64, 112001 (2001).

[11] Z. Maki, M. Nakagawa and S. Sakata, Prog. Theor. Phys. 28, 870 (1962).

[12] K. Hagiwara and N. Okamura, Nucl. Phys. B548, 60 (1999).

[13] M. Aoki, K. Hagiwara, Y. Hayato, T. Kobayashi, T. Nakaya, K. Nishikawa, and N. Okamura, Phys. Rev. D67, 093004 (2003); hep-ph/0104220 M. Aoki, hep-ph/0204008 N. Okamura, hep-ph/0204118.

[14] See the MINOS collaboration's home page, http://www-numi.fnal.gov/

[15] Y. Itow et al., (T2K neutrino Project), hep-ex/0106019; see also the T2K Neutrino Working Group's home page, http://neutrino.kek.jp/jhfnu/

[16] M. Aoki, K. Hagiwara, and N. Okamura, Phys. Lett. B554, 121 (2003); N. Okamura, hep-ph/0209123.

[17] See J-PARC's home page, http://j-parc.jp/.

[18] See e.g. , T. Kobayashi, presented at NP02, Kyoto, Sept. 2002.

[19] M. Aoki, K. Hagiwara, and N. Okamura, in preparation.

[20] See e.g., Y. Kuno et al. http://www-prism.kek.jp/nufactj/index.html.

[21] H. Chen, et al., hep-ph/0104266 Y.F. Wang, et al., Phys. Rev. D65, 073021 (2002). 
[22] Y.F. Wang, hep-ex/0010081.

[23] V. Barger, S. Geer, R. Raja, K. Whisnant, Phys. Lett. B485, 379 (2000) A. Cervera, A. Donini, M.B. Gavela, J.J. Gomez Cadenas, P. Hernandez, O. Mena, S. Rigolin, Nucl. Phys. B579, 17 (2000); Erratum-ibid. Nucl. Phys. B593, 731 (2001); Nucl. Instrum. Meth. A472, 403 (2000); A. Donini, D. Meloni, P. Migliozzi, Nucl. Phys. B646, 321 (2002); Y.F. Wang, K. Whisnant, B.L. Young, Phys. Rev. D65, 073006 (2002); V. Barger, D. Marfatia, K. Whisnant, Phys. Rev. D65, 073023 (2002); P. Huber, M. Lindner, W. Winter, Nucl. Phys. 645, 3 (2002); J. Burguet-Castell, M.B. Gavela, J.J. Gomez-Cadenas, P. Hernandez, O. Mena, Nucl. Phys. B646, 301 (2002).

[24] See e.g. , K. Ishihara, Ph.D. thesis, ICRR-Report-457-2000-1;Y. Hayato, Nucl. Phys. Proc. Suppl.112, 171 (2002).

[25] E. Paschos and J.Y. Yu, Phys. Rev. D65, 033002 (2002).

[26] S. Eidelman et al., Phys. Lett. B592, 1 (2004). 